Nervenarzt 2022 $93: 840-843$ https://doi.org/10.1007/s00115-021-01257-z Angenommen: 3. Dezember 2021 Online publiziert: 10. Januar 2022 (c) Der/die Autor(en) 2022

\section{Alltagsbeeinträchtigungen bei neurokognitiven Störungen}

\section{Entwicklung eines differenzierten Erfassungsinstrumentes für Forschung und Praxis}

\author{
Katja Funke ${ }^{1,2} \cdot$ Marie Bernard ${ }^{3,4} \cdot$ Melanie Luppa $\cdot$ Steffi G. Riedel-Heller ${ }^{5}$. \\ Tobias Luck ${ }^{1,6}$ \\ 'Institut für Sozialmedizin, Rehabilitationswissenschaften und Versorgungsforschung (ISRV) \& \\ Fachbereich Wirtschafts- und Sozial wissenschaften, Hochschule Nordhausen, Nordhausen, Deutschland; \\ ${ }^{2}$ Institut für Gesundheits- und Pflegewissenschaft, Medizinische Fakultät, Martin-Luther-Universität Halle- \\ Wittenberg, Halle (Saale), Deutschland; ${ }^{3}$ Institut für Medizinische Soziologie, Medizinische Fakultät, \\ Martin-Luther-Universität Halle-Wittenberg, Halle (Saale), Deutschland; ${ }^{4}$ SRH Hochschule für \\ Gesundheit, Gera, Deutschland; ${ }^{5}$ Institut für Sozialmedizin, Arbeitsmedizin und Public Health (ISAP), \\ Medizinische Fakultät, Universität Leipzig, Leipzig, Deutschland; ${ }^{6}$ Fakultät Angewandte \\ Sozialwissenschaften, Fachhochschule Erfurt, Erfurt, Deutschland
}

\section{Hintergrund}

Bei der Diagnostik von Demenzen und deren potenziellen Frühformen kommt der sorgfältigen Erfassung, inwieweit betroffene Menschen ihren Alltag noch bzw. nicht mehr bewältigen können, eine enorme Bedeutung zu. So müssen beispielsweise für die Diagnose einer Demenz nach den Kriterien des internationalen Klassifikationssystems International Classification of Diseases-10th Revision (ICD-10) mit einem objektiven kognitiven Abbau einhergehende Beeinträchtigungen von Aktivitäten des täglichen Lebens nachgewiesen werden [4]. Die aktuelle fünfte Version des Klassifikationssystems Diagnostic and Statistical Manual of Mental Disorders (DSM) unterscheidet explizit zwischen einer schweren neurokognitiven Störung - eine Diagnose, die das noch im DSMIV aufgeführte Demenzsyndrom in etwa ersetzt - und einer leichten neurokognitiven Störung, welche in vielen Fällen eine mögliche Vorstufe/frühe Ausprägung einer Demenz darstellen kann [1, 2]. Auch für die Diagnose dieser beiden Formen neurokognitiver Störungen und insbesondere deren Abgrenzung voneinander stellen Beeinträchtigungen in den Alltagsaktivitäten ein entscheidendes Kriterium dar, müssen für die schwere neurokognitive Störung doch eine durch die kognitiven Einschränkungen beeinträchtigte Unabhän- gigkeit in der Verrichtung alltäglicher Aktivitäten nachgewiesen werden, während bei der leichten neurokognitiven Störung die selbständige Verrichtung trotz kleinerer Einschränkungen noch möglich sein sollte [2]. Auch mit der in der neuen 11. Version der ICD-Klassifikation ebenfalls vorgenommenen Aufnahme der Diagnose leichte neurokognitive Störung [5] wird die Bedeutung der sorgfältigen Erfassung von Beeinträchtigungen in Alltagsaktivitäten zunehmen. Trotz dieser enormen Relevanz von Alltagsbeeinträchtigungen wird Diagnostiker/innen bei deren Erfassung erstaunlich viel Spielraum überlassen.

\section{Zielsetzung}

Ziel des hier vorgestellten und von der Deutschen Alzheimer Gesellschaft e.V. - Selbsthilfe Demenz geförderten Forschungsprojektes „Entwicklung eines Instrumentes für die differenzierte Erfassung von Alltagsbeeinträchtigungen aufgrund kognitiver Abbauprozesse Ein Ansatz zur Verbesserung der Früherkennung und Diagnostik von Demenzen und deren Vorstufen leichter neurokognitiver Störungen in Forschung und Praxis" ist die Entwicklung, Evaluation und Bereitstellung eines standardisierten deutschsprachigen Instrumentes für die differenzierte, ökonomische Erfassung von Alltagsbeeinträchtigungen aufgrund 


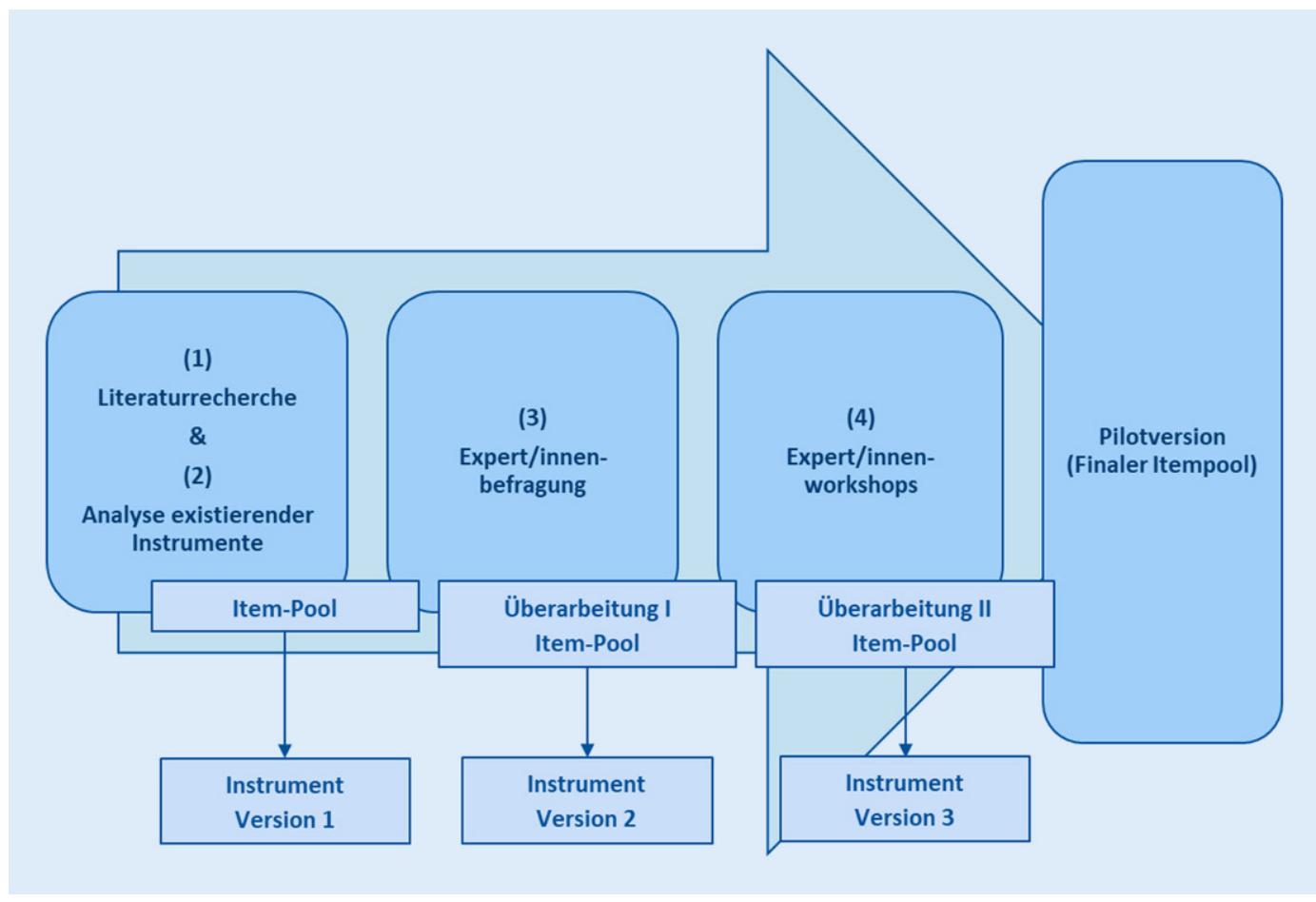

Abb. $1 \varangle$ Stufen der Entwicklung des Erfassungsinstrumentes in Projektphase 1

neurodegenerativer Abbauprozesse. Das Erfassungsinstrument soll eine empirisch begründete Differenzierung von Alltagsbeeinträchtigungen ermöglichen, welche 1. mit leichten neurokognitiven Störungen und schweren neurokognitiven Störungen/Demenzen assoziiert sind (Differenzierung nach dem Schweregrad) und

2. mit Defiziten in spezifischen kognitiven Bereichen assoziiert sind (entsprechend der sechs im DSM-5 differenzierten neurokognitiven Domänen [2]; Differenzierung nach dem Bereich der kognitiven Defizite).

Die Bereitstellung des Erfassungsinstrumentes soll die Früherkennung und (Differenzial-)Diagnostik von Demenzen sowie deren (potenziellen) Vorstufen in Forschung und klinischer Praxis unterstützen und damit zur Verbesserung der Versorgungssituation der Betroffenen selbst sowie auch zu einer Entlastung deren Angehöriger beitragen.

\section{Methodik}

Das Forschungsprojekt wird in Kooperation der Hochschule Nordhausen (Prof. Dr. rer. med. habil. Tobias Luck, Principal Investigator) mit der Universität Leipzig (Prof. Dr. med. Steffi G. Riedel-Heller, MPH, Kooperationspartnerin) durchgeführt und umfasst zwei Projektphasen.

In der an der Hochschule Nordhausen durchgeführten Projektphase 1 (01.03.2020-30.04.2021) erfolgte die Erstellung einer Pilotversion des Erfassungsinstrumentes. Hierfür wurde zunächst in drei sich einander ergänzenden Arbeitsschritten ein umfassender potenzieller Itempool für das Instrument generiert:

1. Literaturrecherche in (inter-)nationalen Fachdatenbanken,

2. Analyse bereits existierender Untersuchungsinstrumente,

3. Durchführung einer Expert/ innenbefragung (standardisierte postalische Befragung von $n=20$ klinisch tätigen Diagnostiker/innen).

Aufbauend auf dem erarbeiteten Itempool erfolgte in einem 4. Arbeitsschritt im Rahmen von acht Expert/innenworkshops die finale Erstellung der Pilotversion des Instrumentes (- Abb. 1). An den Workshops nahmen insgesamt $n=24$ Personen aus vier Gruppen ( $n=6$ Senior/innen, $n=6 \mathrm{An}$ gehörige älterer Menschen mit Demenz, $n=7$ Pflege- und Gesundheitsfachkräfte, $n=5$ Diagnostiker/innen) teil. In den Workshops wurde eine Vorversion des Instrumentes auf Anwendbarkeit, Voll- ständigkeit und Verständlichkeit geprüft. Hierzu wurden von zwei Projektmitarbeiter/innen anhand eines leitfadenbasierten, halbstandardisierten Interviews Fragen zu Instruktion, Items sowie der Antwortskala des Erfassungsinstrumentes gestellt und ausgewertet. Die Durchführung der Workshops erfolgte aufgrund der Corona-Pandemie ausschließlich online per Videokonferenzsystem BigBlueButton (Open-Source-Webkonferenzsystem; https://bigbluebutton.org/).

In der aktuell an der Universität Leipziglaufenden Projektphase 2 (01.03.202131.08.2022) erfolgt die psychometrische Evaluierung und Finalisierung des Erfassungsinstrumentes im Rahmen einer Pilotstudie mit insgesamt $n=90$ Proband/ innen (60+ Jahre) unterteilt in drei Gruppen: $n=30$ Proband/innen ohne kognitive Defizite, $n=30$ Proband/innen mit leichten neurokognitiven Störungen sowie $n=30$ Proband/innen mit klinisch manifester Demenz. Detaillierte Ziele der Pilotstudie sind neben der Überprüfung der Verständlichkeit und Klarheit des Erfassungsinstrumentes die Überprüfung der psychometrischen Gütekriterien [3] und eine weitere Reduktion der Itemanzahl.

Alle beschriebenen Befragungen und Untersuchungen von Studienteilnehmer/ innen erfolgten bzw. erfolgen mit Zustim- 


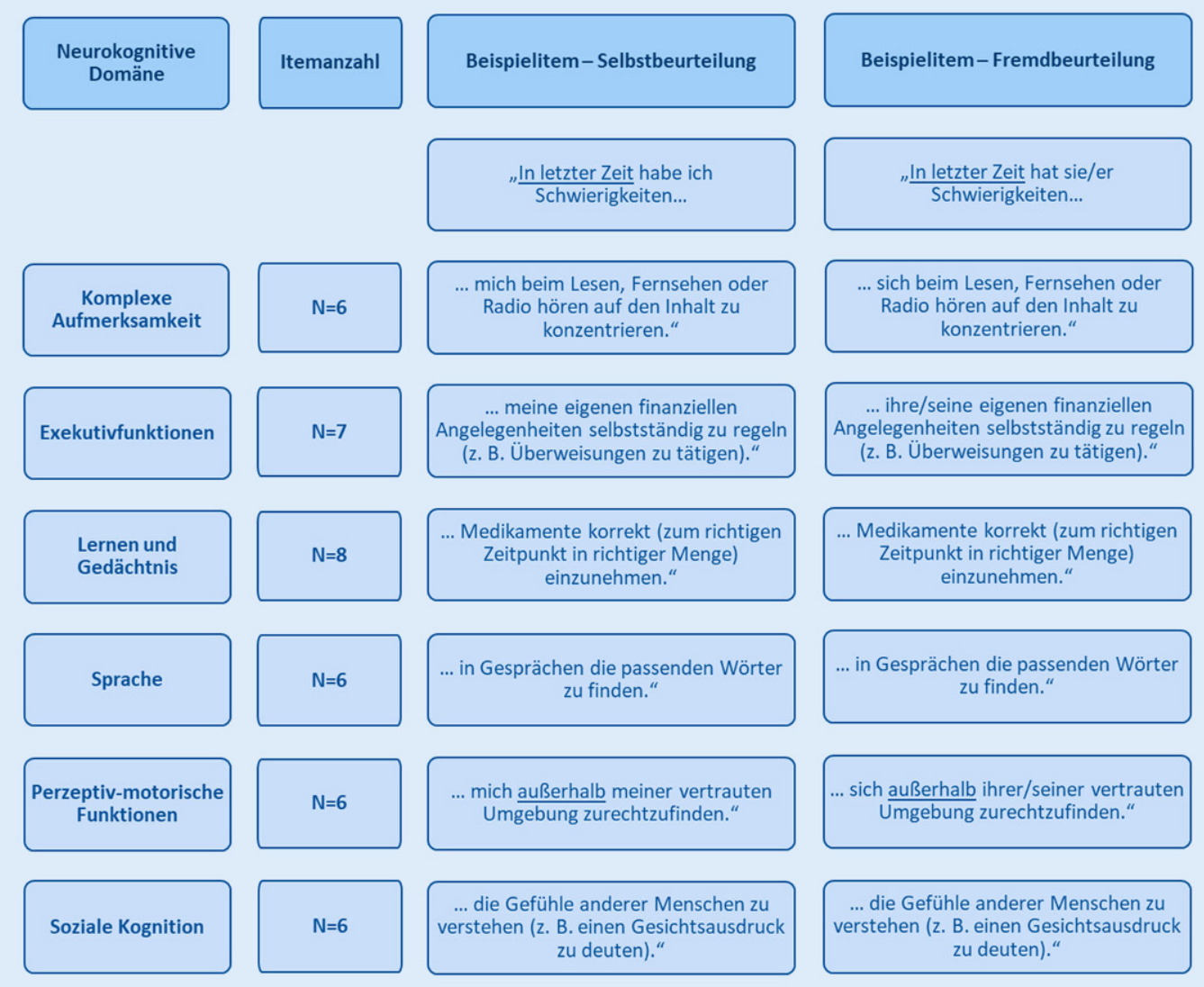

Abb. $2 \triangleleft$ Aufbau der Pilotversion des Instrumentes für die Erfassung von Alltagsbeeinträchtigungen bei neurokognitiven Störungen (A-NKS)

mung der zuständigen Ethikkommissionen (Projektphase 1: Ethikkommission des Universitätsklinikums Jena, AZ 2020-1709Bef \& 2020-1709_1-Bef; Projektphase 2: Ethikkommission an der Medizinischen Fakultät der Universität Leipzig, AZ 217/21ek) im Einklang mit nationalem Recht sowie gemäß der Deklaration von Helsinki von 1975 (in der aktuellen, überarbeiteten Fassung; [6]). Von allen beteiligten Studienteilnehmer/innen liegt eine Einverständniserklärung vor bzw. wird eine Einverständniserklärung vor Teilnahme eingeholt.

\section{Aktuelle Ergebnisse}

Mit dem Abschluss der Projektphase 1 konnte eine Pilotversion des Instrumentes für die Erfassung von Alltagsbeeinträchtigungen bei neurokognitiven Störungen (A-NKS) erstellt werden, die aktuell in Projektphase 2 evaluiert wird. Die Pilotversion wurde hierbei sowohl als Selbst- (A-NKSSB) als auch als Fremdbeurteilungsversion (A-NKS-FB) erstellt. Beide Versionen umfassen jeweils 39 Items, die, den Zielen des Forschungsprojektes entsprechend, so zusammengestellt wurden, dass sie Beeinträchtigungen in Alltagsaktivitäten erfassen sollen, die Defiziten in den vom DSM-5 differenzierten sechs neurokognitiven Domänen [2] spezifisch zugeordnet werden können. Einen Überblick über die neurokognitiven Domänen des DSM-5, die Anzahl der jeweils in der erstellten Pilotversion des Erfassungsinstrumentes zugeordneten Items sowie Beispielitems aus Selbst- und Fremdbeurteilungsversion gibt - Abb. 2. Zur Beurteilung des Vorliegens der Alltagsbeeinträchtigungen wurde in den beiden Versionen allen Items eine Antwortskala zugeordnet (keine Schwierigkeiten - leichte Schwierigkeiten - mittlere Schwierigkeiten - große Schwierigkeiten - Es gelingt mir/ihr/ihm gar nicht - Trifft auf mich/sie/ihn nicht zu), welche zusätzlich eine Differenzierung nach dem Schweregrad einer potenziell vorliegenden neurokognitiven Störung/ Demenz erlauben soll.

\section{Ausblick}

Nach erfolgreicher Evaluierung und Finalisierung der Pilotversion des Erfassungsinstrumentes in Projektphase 2 wird die finale Version einem breiten Anwender/innenkreis in Forschung und Praxis (Hausärzt/innen, Fachärzt/innen, Neuropsycholog/innen etc.). kostenfrei zur Verfügung gestellt.

\section{Fazit für die Praxis}

- Es wird ein Erhebungsinstrument entwickelt und kostenfrei zur Verfügung gestellt, welches eine Erfassung von Alltagsbeeinträchtigungen bei neurokognitiven Störungen differenziert nach dem Schweregrad (leichte vs. schwere neurokognitive Störung/Demenz) und nach dem Bereich der kognitiven Defizite (entsprechend den sechs neurokognitiven Domänen des DSM-5) ermöglicht.

- Das Instrument soll so die Früherkennung und (Differenzial-)Diagnostik von Demenzen sowie deren (potenziellen) Vorstufen in Forschung und klinischer Praxis unterstützen. 


\section{Korrespondenzadresse}

\section{Prof. Dr. rer. med. habil. Tobias Luck}

Fakultät Angewandte Sozialwissenschaften, Fachhochschule Erfurt

Altonaer Str. 25, 99085 Erfurt, Deutschland tobias.luck@fh-erfurt.de

Funding. Das Forschungsprojekt wird durch die Deutsche Alzheimer Gesellschaft e. V. - Selbsthilfe Demenz gefördert (Forschungsförderung 2019). Open Access funding enabled and organized by Projekt DEAL.

Interessenkonflikt. K. Funke, M. Bernard, M. Luppa, S.G. Riedel-Heller und T. Luck geben an, dass kein Interessenkonflikt besteht.

Open Access. Dieser Artikel wird unter der Creative Commons Namensnennung 4.0 International Lizenz veröffentlicht, welche die Nutzung, Vervielfältigung, Bearbeitung, Verbreitung und Wiedergabe in jeglichem Medium und Format erlaubt, sofern Sie den/die ursprünglichen Autor(en) und die Quelle ordnungsgemäß nennen, einen Link zur Creative Commons Lizenz beifügen und angeben, ob Änderungen vorgenommen wurden.

Die in diesem Artikel enthaltenen Bilder und sonstiges Drittmaterial unterliegen ebenfalls der genannten Creative Commons Lizenz, sofern sich aus der Abbildungslegende nichts anderes ergibt. Sofern das betreffende Material nicht unter der genannten Creative Commons Lizenz steht und die betreffende Handlung nicht nach gesetzlichen Vorschriften erlaubt ist, ist für die oben aufgeführten Weiterverwendungen des Materials die Einwilligung des jeweiligen Rechteinhabers einzuholen.

Weitere Details zur Lizenz entnehmen Sie bitte der Lizenzinformation auf http://creativecommons.org/ licenses/by/4.0/deed.de.

\section{Literatur}

1. American Psychiatric Association (2009) Diagnostic and statistical manual of mental disorders. DSM-IV-TR, 4. Aufl. American Psychiatric Assoc, Arlington (text revision, 13. print)

2. American Psychiatric Association (2013) Diagnos tic and statistical manual of mental disorders. DSM-5, 5. Aufl. American Psychiatric Publishing, Washington, DC

3. Bühner M (2011) Einführung in die Test- und Fragebogenkonstruktion, 3. Aufl. PS Psychologie. Pearson Studium, München

4. Dilling $\mathrm{H}$, Mombour W, Schmidt MH et al (Hrsg) (2015) Internationale Klassifikation psychischer Störungen. ICD-10 Kapitel V (F) klinisch-diagnostische Leitlinien, 10. Aufl. Hogrefe, Bern (unter Berücksichtigung der Änderungen entsprechend ICD-10-GM 2015)

5. Jessen F, Frölich L (2018) ICD-11: Neurokognitive Störungen. Fortschr Neurol Psychiatr 86(3):172-177

6. Wiesing U, Ehni H-J (2014) Die Deklaration von Helsinki des Weltärztebundes - Ethische
Grundsätze für die Forschung am Menschen. In Lenk C, Duttge G, Fangerau H (Hrsg) Handbuch Ethik und Recht der Forschung am Menschen. Springer, Berlin, Heidelberg, S517-524

\section{Hier steht eine Anzeige.}

\section{Springer}

